\title{
Coercive Journal Self Citations, Impact Factor, Journal Influence and Article Influence
}

\author{
Chia-Lin Chang \\ Department of Applied Economics \\ Department of Finance \\ National Chung Hsing University \\ Taichung, Taiwan \\ Michael McAleer \\ Econometric Institute \\ Erasmus School of Economics \\ Erasmus University Rotterdam \\ and \\ Tinbergen Institute \\ The Netherlands \\ and \\ Department of Quantitative Economics \\ Complutense University of Madrid \\ and \\ Institute of Economic Research \\ Kyoto University \\ Les Oxley \\ Department of Economics \\ University of Waikato \\ New Zealand
}

Revised: March 2013 


\begin{abstract}
This paper examines the issue of coercive journal self citations and the practical usefulness of two recent journal performance metrics, namely the Eigenfactor score, which may be interpreted as measuring “Journal Influence”, and the Article Influence score, using the Thomson Reuters ISI Web of Science (hereafter ISI) data for 2009 for the 200 most highly cited journals in each of the Sciences and Social Sciences. The paper also compares the two new bibliometric measures with two existing ISI metrics, namely Total Citations and the 5year Impact Factor (5YIF) (including journal self citations) of a journal. It is shown that the Sciences and Social Sciences are different in terms of the strength of the relationship of journal performance metrics, although the actual relationships are very similar. Moreover, the journal influence and article influence journal performance metrics are shown to be closely related empirically to the two existing ISI metrics, and hence add little in practical usefulness to what is already known, except for eliminating the pressure arising from coercive journal self citations. These empirical results are compared with existing results in the bibliometrics literature.
\end{abstract}

Keywords: Journal performance metrics, Coercive journal self citations, Research assessment measures, Total citations, 5-year impact factor (5YIF), Eigenfactor, Journal influence, Article influence.

JEL Classification: A12. 


\section{“They're digging in the wrong place!" Indiana Jones, Raiders of the Lost Ark}

\section{Introduction}

Evaluating research quality is fundamental to the Sciences and Social Sciences. Research assessment rankings are essential to evaluate the research performance of individuals and the quality of academic journals. The perceived research performance of individual researchers is crucial for hiring, firing, tenure and promotion decisions. In the absence of clear signals regarding the inherent, and frequently latent, quality of published research, the perceived quality of a journal is frequently used as a proxy, albeit inappropriately, for the quality of a research paper, This situations arises especially in the Social Sciences, where citations to published papers do not seem to be as widely used as they are in the Sciences.

Most journal performance metrics are based on alternative transformations of citations and journal influence. The Thomson Reuters ISI Web of Science database [16] (hereafter ISI) is a leading high quality database for generating research assessment measures, especially citations, to evaluate the research performance of individual researchers and the quality of academic journals. Although there are caveats regarding the methodology and data collection methods underlying any database (see, for example, Seglen [18], Chang and McAleer [6, 7], Chang, McAleer and Oxley [8, 9, 10, 11] for caveats regarding ISI), the ISI citations database is the oldest source of rankings criteria and the benchmark against which other databases are compared.

This paper examines the issue of coercive journal self citations, and the practical usefulness of two new journal performance metrics, namely the Eigenfactor score, which may be interpreted as measuring “Journal Influence”, and Article Influence score, using ISI data for 2009 for the 200 most highly cited journals in each of the Sciences and Social Sciences. The paper also compares the two new bibliometric measures with two existing ISI metrics, namely Total Citations and the 5-year Impact Factor (5YIF) (including journal self citations) of a journal. 
It is shown that the Sciences and Social Sciences are different in terms of the strength of the relationship of journal performance metrics, although the actual relationships are nevertheless very similar. Moreover, the Journal Influence and Article Influence metrics are shown to be closely related empirically to the two existing ISI metrics, so that they add little to what is already known about journal impact, except for eliminating the pressure arising from coercive journal self citations. These empirical results are compared with existing results in the literature.

The plan of the remainder of the paper is as follows. Section 2 presents three key research assessment measures (RAM), namely the 5-year impact factor (5YIF) (including journal self citations) of a journal, Eigenfactor (or Journal Influence) score, and Article Influence score, and discusses the incidence of coercive journal self citations in several disciplines. Section 3 reports some empirical analyses of these key RAM, as well as Total Citations, and compares the results with those that are available in the literature. Section 4 gives some concluding remarks.

\section{Key Research Assessment Measures (RAM)}

It is well known that coercive journal citations by journal editors and journal publishers can have a deleterious and misleading impact on journal self citations. Wilhite and Fong [19, p. 542] find, on the basis of "6,672 responses from a survey sent to researchers in economics, sociology, psychology, and multiple business disciplines (marketing, management, finance, information systems, and accounting), as well as data from 832 journals in those same disciplines" that "coercion is uncomfortably common and appears to be practiced opportunistically”. In short, many journal editors in these disciplines would seem to be encouraging to increase journal self citations or risk rejection. Wilhite and Fong [19, p. 543] also find that "Coercive self-citation exists and is more common in the business disciplines than in economics, sociology, and psychology.”

The classic 2-year impact factor including journal self citations (2YIF) of a journal is typically referred to as "the impact factor". An analysis of the 2-year impact factors reported for the ISI Economics category in Chang, Maasoumi and McAleer [5] suggested that the 
inflated impact factors were rather high, with mean impact factor inflation of 1.442, and with 9 of 299 Economics journals having impact factors inflated by a factor of at least 3. This would seem to suggest that self citations in a large number of journals in the Economics category are consistent with coercion.

It is worth emphasizing that coercive journal self citation practices of editors and publishers seem to be concentrated on the 2-year impact factor than the 5-year impact factor. The use of both two and five years for the analysis of journal impact is entirely arbitrary. Although it is not entirely clear why this might be the case, it is worth venturing that the 2-year impact factor is more widely used and known than its 5-year counterpart. Therefore, the 2-year impact factor is flawed in the sense that its calculation suffers from the well known coercive journal self citations, as discussed above. However, even if the most recent two years of the 5-year impact factor are affected by coercive journal self citations, as is likely, the first three years of the 5-year impact factor are unlikely to have been as distorted by coercive journal self citations. In this sense, the 5-year impact factor is likely to be more accurate in capturing citations without being skewed by coercive journal self citations.

With this in mind, three leading journal performance measures, one of which includes journal self citations while the other two do not, for an ISI Journal Citations Reports (JCR) calendar year, which is the year before the annual Research Assessment Measures (RAM) are released, are as follows:

\section{(1) 5-year impact factor including journal self citations (5YIF):}

In a similar manner to the classic 2-year impact factor, the 5-year impact factor including journal self citations (5YIF) of a journal is calculated annually, and is defined as "Total citations in a year to papers published in a journal in the previous 5 years / Total papers published in a journal in the previous 5 years.” The choice of 5 years by ISI is arbitrary. Although 5YIF is not widely reported, it is widely held in the academic community, and certainly by the editors and publishers of journals, that a higher 5YIF is better than lower. It is worth noting that, for whatever reason, 5-year impact factor excluding journal self citations is not presently calculated by ISI [16].

\section{(2) Eigenfactor (or Journal Influence) score:}


The Eigenfactor score (see Bergstrom [2], Bergstrom and West [3], Bergstrom, West and Wiseman [4]) is calculated annually (see www.eigenfactor.org), and is defined as: "The Eigenfactor Score calculation is based on the number of times articles from the journal published in the past five years have been cited in the JCR year, but it also considers which journals have contributed these citations so that highly cited journals will influence the network more than lesser cited journals. References from one article in a journal to another article from the same journal are removed, so that Eigenfactor Scores are not influenced by journal self-citation." Unfortunately, there is no indication as to the value of the threshold that separates "highly cited" from "lesser cited” journals, or how the former might "influence the network more" than the latter. Even though Eigenfactor does not check how much time researchers spend reading hard copies of journals, which would require extensive surveys across a wide range of disciplines, it does indicate how much time researchers might spend reading or scanning articles on a journal's website. Thus, Eigenfactor might usefully be interpreted as a "Journal Influence” measure (see Chang, Maasoumi and McAleer [5]). A higher Eigenfactor score would be preferred to lower.

\section{(3) Article Influence:}

Article Influence (see Bergstrom [2], Bergstrom and West [3], Bergstrom, West and Wiseman [4]) measures the relative importance of a journal's citation influence on a perarticle basis. Despite the misleading suggestion of measuring “Article Influence”, as every journal has only one Article Influence score, this RAM is actually a "per capita Journal Influence” score. Article Influence is a standardized Eigenfactor score, is calculated annually, and is defined as "Eigenfactor score divided by the fraction of all articles published by a journal.” A higher Article Influence would be preferred to lower.

\section{Empirical Analysis}

\subsection{Existing empirical results}


Davis [12] used two simple linear regressions to relate the logarithm of the Eigenfactor score to the logarithm of Total Citations, and the logarithm of 2YIF to the logarithm of the Eigenfactor score, giving a high $R^{2}=0.950$ and a reasonably high $R^{2}=0.860$, respectively. These are interesting empirical findings, even though it might be argued that the results would have been more informative if the Eigenfactor score had been related to 5YIF as both bibliometric measures are calculated over a 5-year citation period, although they are defined differently.

Using a simple linear regression in levels rather than logarithms, Fersht [14] showed that there was a high $R^{2}=0.968$ between the Eigenfactor score and Total Citations for the top 200 most highly cited ISI journals (based on 2YIF) in the Sciences, based on ISI Total Citations data for 2007. This is very similar to the results obtained for the Eigenfactor score and Total Citations in Davis [12], even though the simple linear regressions in the two papers differed in terms of the data transformations used.

Rousseau et al. [17] calculated the Spearman correlation coefficients between the pairs 2YIF and Eigenfactor score, 2YIF and Article Influence score, and Eigenfactor and Article Influence scores to be $0.827,0.918$ and 0.827 , respectively. It might be argued that these interesting empirical results might have been more relevant if 5YIF had been related to the Eigenfactor and Article Influence scores as each of these three bibliometric measures is calculated over a 5-year citation period.

Franceschet [15] considered three pairs of variables for calculating correlation coefficients, namely 2YIF and Eigenfactor score, 5YIF and Eigenfactor score, and the Eigenfactor and Article Influence scores, giving correlations of $0.770,0.770$ and 0.760 , respectively. Two simple linear regressions in the levels of three RAM were considered, with $R^{2}=0.810$ for a simple linear regression of Article Influence score on 2YIF, and $R^{2}=0.880$ for a simple linear regression of Article Influence score on 5YIF. The marginal effects of 2YIF and 5YIF on the Article Influence score were 0.446 and 0.452 , respectively.

Elkins et al. [13] and Arendt [1] both considered the relationship between 2YIF and Article Influence score, with the former calculating a correlation coefficient of 0.790 and the latter a relatively low $R^{2}=0.596$ from a simple linear regression based on the median values across 
a range of scientific fields. It might be repeated that these interesting empirical results would have been more meaningful if the Article Influence score had been related to 5YIF rather than 2YIF so that the bibliometric measures would have been calculated over the same citation period.

\subsection{Additional empirical results}

In order to contribute to the existing literature on empirical findings regarding alternative RAM, in Figures 1-4 we evaluate the 200 most highly cited journals, according to 2YIF, in both the sciences and social sciences for 2009. These figures relate the Eigenfactor score to Total Citations and the Article Influence score to 5YIF. The Eigenfactor score (or Journal Influence) is an aggregate figure, as is Total Citations, whereas both the Article Influence score and 5YIF are ratios.

The Total Citations data for 2009 for the Sciences and Social Sciences, respectively, were downloaded from ISI on 19 June 2010 and 20 June 2010.

A simple linear regression, with the Eigenfactor score (or Journal Influence) as a function of Total Citations, is given in Figures 1 and 3 for the Sciences and Social Sciences, respectively. The empirical analysis is seeking to explain Journal Influence as a function of Total Citations to examine if the volume of citations affects the influence of a journal.

The estimated regression model shows that the Eigenfactor score (or Journal Influence) increases, on average, by 0.000004 and 0.000003 for each unit increase in Total Citations for 2009 for the Sciences and Social Sciences, respectively. The goodness-of-fit measures, namely $R^{2}=0.931$ and $R^{2}=0.659$ for the Sciences and Social Sciences, respectively, show that the Eigenfactor score can be estimated quite accurately, especially for the Sciences, on the basis of a simple linear regression against Total Citations.

The approximate relationships between the Eigenfactor score (or Journal Influence) and Total Citations for the Sciences and Social Sciences, respectively, can be expressed as:

Eigenfactor score $=k$ (Total Citations) 
where $k=0.0000033$ and $k=0.000002$ for Sciences and Social Sciences, respectively. The estimated value of $k=0.00000396$ in Ferscht [14] for the Sciences, based on ISI Total Citations data for 2007, is broadly similar to the result obtained in the present paper, as is the value of $R^{2}$.

It is worth noting that the Eigenfactor score (or Journal Influence) does not suffer from coercive journal self citations. However, Total Citations do include coercive journal self citations, though it is not known to what extent. It is, therefore, interesting that these two bibliometric measures are so highly correlated.

Another simple linear regression, with the Article Influence score as a function of 5YIF, is given in Figures 2 and 4 for 2009 for the Sciences and Social Sciences, respectively. The empirical analysis is seeking to explain Article Influence as a function of 5YIF to examine if the impact factor calculated over a five-year period affects the influence of a journal on a per capita article basis.

The estimated models show that the Article Influence score increases, on average, by 0.489 and 0.479 for each unit increase in 5YIF for 2009 for the Sciences and Social Sciences, respectively.

The goodness-of-fit measures, as given by $R^{2}=0.923$ and $R^{2}=0.572$ for 2009 for the Sciences and Social Sciences, respectively, show that the Article Influence score can be approximated very accurately for the Sciences, and reasonably accurately for the Social Sciences, on the basis of a simple linear regression relationship of Article Influence score against 5YIF, namely:

Article Influence score $=5 Y I F / 2$.

Although the goodness-of-fit value of $R^{2}$ obtained in the present paper is slightly higher than in Franceschet (2009), namely $R^{2}=0.880$, in relating the Article Influence score to 5YIF, the latter paper had an effect of 5YIF on Article Influence score of 0.452 , which is very similar to that proposed above. 
It is worth noting that the Article Influence score does not suffer from coercive journal self citations, whereas 5YIF does include coercive journal self citations, though it is not known to what extent. The coercive journal self citations contained in 5YIF would most likely be greatest in the most recent two years, as 2YIF is known to be affected by coercive journal self citations. It is, therefore, interesting that these two bibliometric measures are so highly correlated.

\section{Conclusion}

Although the Sciences and Social Sciences are dramatically different in terms of the strength of the underlying relationship of the journal performance metrics considered in this paper, the actual empirical relationships are broadly similar. As both Article Influence and 5YIF are measured over a five-year period, although based on different definitions, it is perhaps not altogether surprising that the two scores are highly and positively correlated.

Given the very high correlations between the Eigenfactor score (or Journal Influence) and Total Citations, and between the Article Influence score and 5YIF, and the corresponding high $R^{2}$ values for the simple linear regressions, the Eigenfactor score and Article Influence score would not seem to be entirely necessary for the Social Sciences, and not at all necessary for the Sciences, relative to the leading journal performance measures that are already available, namely Total Citations and 5YIF, respectively. This statement holds in spite of the fact that neither the Eigenfactor score (or Journal Influence) or Article Influence score suffers from coercive journal self citations, whereas Total Citations and 5YIF are affected by coercive journal self citations, though to unknown degrees.

Ideally, ISI [16] will in the future report a 5-year impact factor excluding journal self citations, as ISI already reports a 2-year impact factor excluding journal self citations. However, as impact factors that exclude journal self citations are necessarily lower than impact factors that include journal self citations, whether coercive or not, journal editors and journal publishers are unlikely to advertise the former. 
As the journal performance measures captured in the Eigenfactor score and Article Influence score, which are said to measure "importance" and "prestige", respectively, add little to what is already available in the ISI Total Citations and 5-year impact factor (5YIF) of a journal, we have no hesitation in concurring with Indiana Jones, who made the following remark regarding his competitors who were searching for the Lost Ark of the Covenant: "They're digging in the wrong place!”

\section{Acknowledgements}

The authors are grateful to two referees for helpful comments and suggestions. For financial support, the first author acknowledges the National Science Council, Taiwan; the second author acknowledges the Australian Research Council, National Science Council, Taiwan, and the Japan Society for the Promotion of Science; and the third author acknowledges the Royal Society of New Zealand Marsden Fund. 


\section{References}

[1] J. Arendt, Are article influence scores comparable across scientific fields?, Issues in Science and Technology Librarianship, (2010) 60. Retrieved from http://www.istl.org/10winter/refereed2.html.

[2] C. Bergstrom, Eigenfactor: Measuring the value and prestige of scholarly journals, C\&RL News, 68 (2007) 314-316.

[3] C.T. Bergstrom, J.D. West, Assessing citations with the Eigenfactor ${ }^{\mathrm{TM}}$ metrics, Neurology, 71 (2008) 1850-1851.

[4] C.T. Bergstrom, J.D. West, M.A. Wiseman, The Eigenfactor ${ }^{\mathrm{TM}}$ metrics, Journal of Neuroscience, 28(45) (2008) 11433-11434.

[5] C.-L. Chang, E. Maasoumi, M. McAleer, Robust ranking of journal quality: An application to economics, (2012) Emory Economics 1204, Department of Economics, Emory University, USA.

[6] C.-L. Chang, M. McAleer, Citations and impact of ISI tourism and hospitality journals, Tourism Management Perspectives, 1(1) (2012) 2-8.

[7] C.-L. Chang, M. McAleer, Ranking journal quality by harmonic mean of ranks: An application to ISI Statistics \& Probability, Statistica Neerlandica, 67(1) (2013) 27-53.

[8] C.-L. Chang, M. McAleer, L. Oxley, What makes a great journal great in economics? The singer not the song, Journal of Economic Surveys, 25(2) (2011a) 326-361.

[9] C.-L. Chang, M. McAleer, L. Oxley, What makes a great journal great in the sciences? Which came first, the chicken or the egg?, Scientometrics, 87(1) (2011b) 17-40.

[10] C.-L. Chang, M. McAleer, L. Oxley, Great expectatrics: Great papers, great journals, great econometrics, Econometric Reviews, 30(6) (2011c) 583-619.

[11] C.-L. Chang, M. McAleer, L. Oxley, How are journal impact, prestige and article influence related? An application to neuroscience, Journal of Applied Statistics, 38(11) (2011d) 2563-2573.

[12] P.M. Davis, Eigenfactor: Does the principle of repeated improvement result in better estimates than raw citation counts?, Journal of the American Society for Information Science and Technology, 59(13) (2008) 2186-2188.

[13] M.R. Elkins, C.G. Maher, R.D. Herbert, A.M. Moseley, C. Sherrington, Correlation between the journal impact factor and three other journal citation indices, Scientometrics, 85 (2010) 81-93. 
[14] A. Fersht, The most influential journals: Impact factor and Eigenfactor, Proceedings of the National Academy of Sciences of the United States of America, 106(17) (2009) 68836884.

[15] M. Franceschet, Journal influence factors, Journal of Informetrics, 4 (2010) 239-248.

[16] ISI Web of Science, Journal Citation Reports, Essential Science Indicators, Thomson Reuters ISI (2010).

[17] R. Rousseau et al., On the relation between the WoS impact factor, the Eigenfactor, the SCImago journal rank, the article influence score and the journal h-index, Conference Proceedings, Nanjing University, April 2009.

[18] P.O. Seglen, Why the impact factor of journals should not be used for evaluating research, BMJ: British Medical Journal, 314(7079) (1997) 498-502.

[19] A.W. Wilhite, E.A. Fong, Coercive citation in academic publishing, Science, 335 (6068), (2012) 542-543. 
Table 1

Correlations and $R^{2}$ for Various Research Assessment Measures (RAM)

\begin{tabular}{|c|c|c|c|}
\hline Authors & Correlated Variables & Correlation & $R^{2}$ \\
\hline \multirow{2}{*}{ Davis [12] } & (log Eigenfactor, log TC) & - & 0.950 \\
\hline & (log 2YIF, log Eigenfactor) & - & 0.860 \\
\hline Ferscht [14] & (Eigenfactor, TC) & - & 0.968 \\
\hline \multirow{3}{*}{ Rousseau et al. [17] } & (2YIF, Eigenfactor) & 0.827 & - \\
\hline & (2YIF, AI) & 0.918 & - \\
\hline & (Eigenfactor, AI) & 0.827 & - \\
\hline \multirow{5}{*}{ Franceschet [15] } & (2YIF, AI) & - & 0.810 \\
\hline & (5YIF, AI) & - & 0.880 \\
\hline & (2YIF, Eigenfactor) & 0.770 & - \\
\hline & (5YIF, Eigenfactor) & 0.770 & - \\
\hline & (Eigenfactor, AI) & 0.760 & - \\
\hline Elkins et al. [13] & (2YIF, AI) & 0.790 & - \\
\hline Arendt [1] & (2YIF, AI) & - & 0.596 \\
\hline \multirow{2}{*}{ This paper } & (Eigenfactor, TC) & - & 0.931 \\
\hline & (5YIF, AI) & - & 0.923 \\
\hline
\end{tabular}

Note: AI denotes Article Influence and TC denotes Total Citations. The correlations are Spearman's correlation coefficients, and the $R^{2}$ values are calculated from simple linear regression models. 
Figure 1

Eigenfactor Score and Total Citations for 200 Most Highly Cited Journals in Sciences for 2009

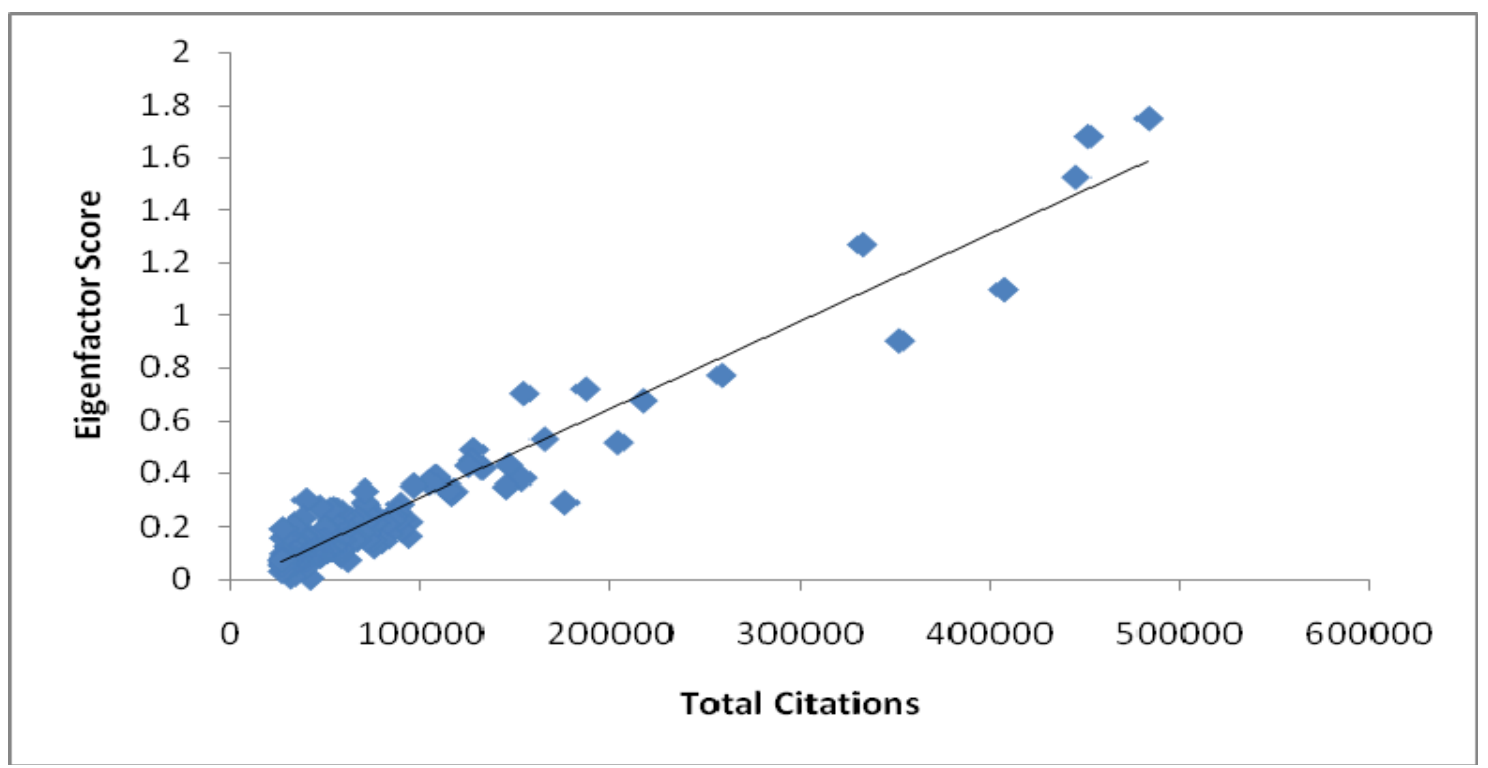

Note: Citations data were downloaded from ISI on 19 June 2010. The OLS regression results are as follows (t-ratios in parentheses):

Eigenfactor Score $=-0.022+3.32 \mathrm{E}-06 \times$ Total Citations + error, $\quad R^{2}=0.931$ $(-3.42)(51.59)$ 
Figure 2

Article Influence Score and 5YIF for 200 Most Highly Cited Journals in Sciences for 2009

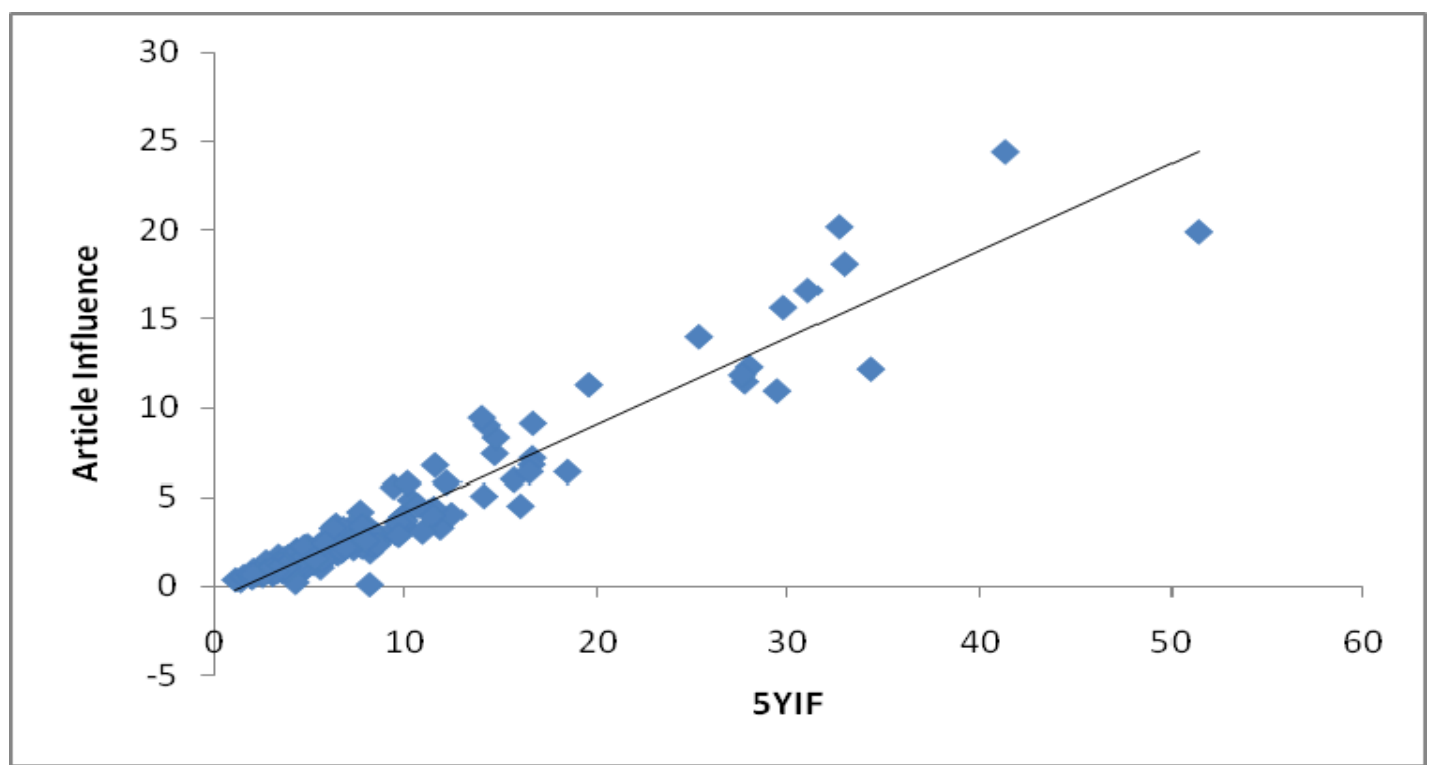

Note: Citations data were downloaded from ISI on 19 June 2010. The OLS regression results are as follows (t-ratios in parentheses):

Article Influence $=-0.719+0.489 \times 5 \mathrm{YIF}+$ error,$\quad R^{2}=0.923$ $(-6.65).(48.54)$ 


\section{Figure 3}

Eigenfactor Score and Total Citations for 200 Most Highly Cited Journals in Social Sciences for 2009

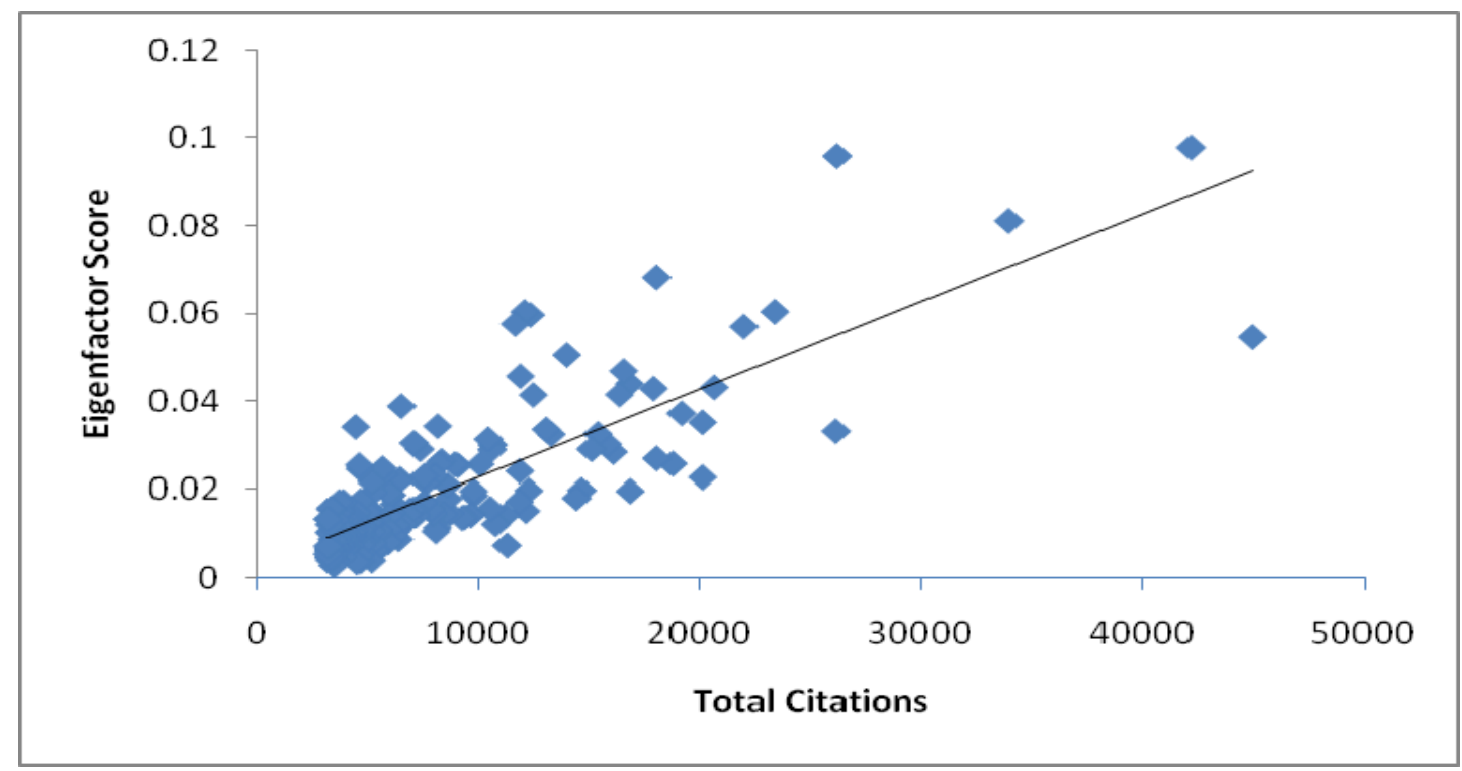

Note: Citations data were downloaded from ISI on 20 June 2010. The OLS regression results are as follows (t-ratios in parentheses):

Eigenfactor Score $=0.029+1.99 \mathrm{E}-06 \times$ Total Citations + error, $\quad R^{2}=0.659$ (2.85) (19.55) 
Figure 4

Article Influence Score and 5YIF for 200 Most Highly Cited Journals in Social Sciences for 2009

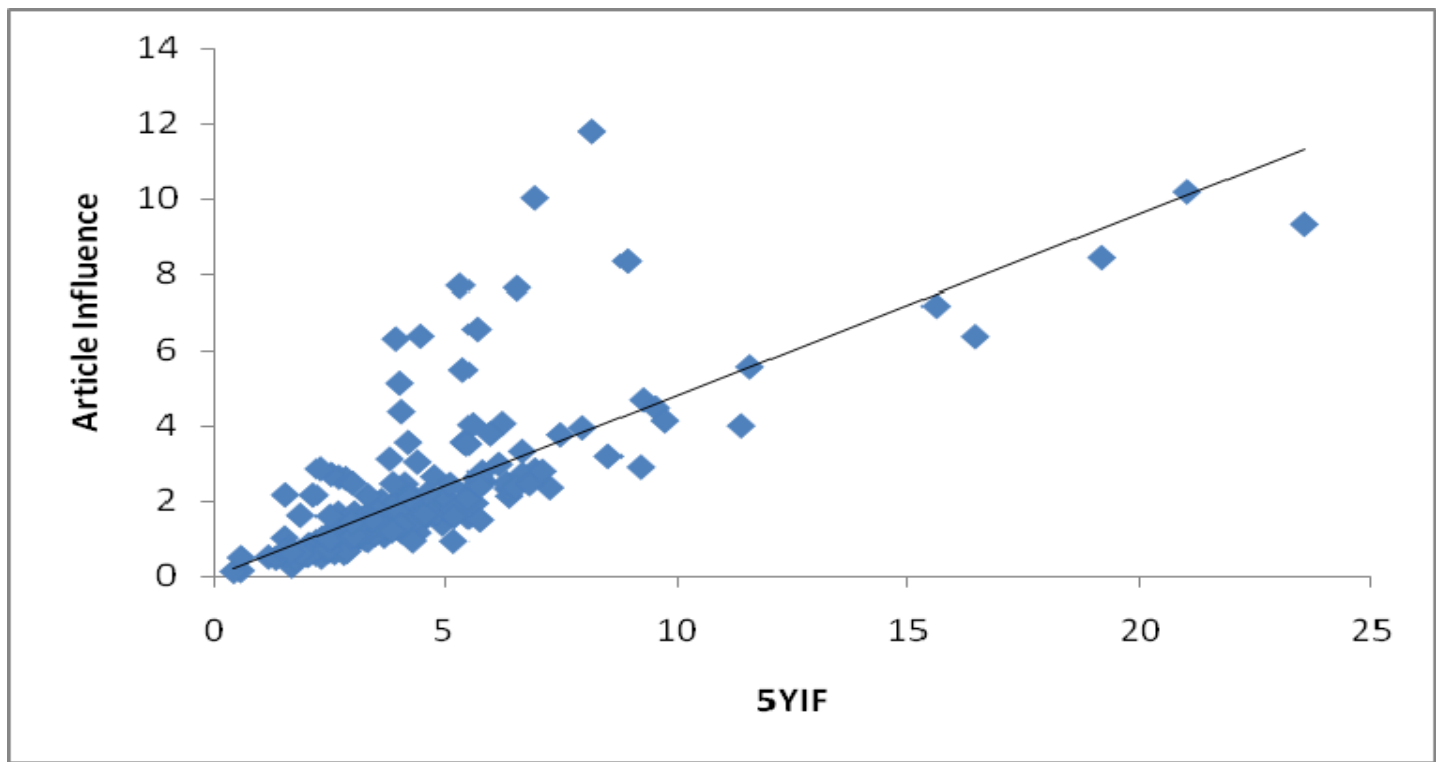

Note: Citations data were downloaded from ISI on 20 June 2010. The OLS regression results are as follows (t-ratios in parentheses):

Article Influence $=0.160+0.479 \times 5 \mathrm{YIF}+$ error,$\quad R^{2}=0.572$

(0.10) (16.25) 